\title{
The integration of auditory and visual motion signals at threshold
}

\author{
S. M. WUERGER \\ Keele University, Keele, England \\ M. HOFBAUER \\ Ludwig-Maximilians-Universität, Munich, Germany \\ and \\ G. F. MEYER \\ Keele University, Keele, England
}

\begin{abstract}
To interpret our environment, we integrate information from all our senses. For moving objects, auditory and visual motion signals are correlated and provide information about the speed and the direction of the moving object. We investigated at what level the auditory and the visual modalities interact and whether the human brain integrates only motion signals that are ecologicallyvalid. We found that the sensitivity for identifying motion was improved when motion signals were provided in both modalities. This improvement in sensitivity can be explained by probability summation. That is, auditory and visual stimuli are combined at a decision level, after the stimuli have been processed independently in the auditory and the visual pathways. Furthermore, this integration is direction blind and is not restricted to ecologically valid motion signals.
\end{abstract}

Single modalities often can represent only partial or ambiguous information about the natural environment. The integration of multisensory information, on the basis that perceptually relevant objects are likely to stimulate multiple sensory systems in parallel, is, therefore, likely to provide more accurate information than single modalities could. Physiological and behavioral studies have provided considerable evidence for the integration of cues across modalities (Stein \& Meredith, 1993). The anatomical structure that is most likely to mediate cross-modal integration of positional cues is the superior colliculus (SC; Gitelman, Parrish, Friston, \& Mesulam, 2002; Meredith, Nemitz, \& Stein, 1987), although recent neurophysiological studies have shown that other cortical areas play a crucial role in forming cross-modal associations (Bremmer, Schlack, Duhamel, Graf, \& Fink, 2001; Bremmer, Schlack, Shah, et al., 2001; Calvert et al., 1999; Calvert et al., 1997; Daffner et al., 2000; Foxe et al., 2000; Foxe \& Simpson, 2002; Fu et al., 2001; Fuster, Bodner, \& Kroger, 2000; Griffiths et al., 1998; King \& Calvert, 2001; Lewis, Beauchamp, \& DeYoe, 2000; Mesulam, Nobre, Kim, Parrish, \& Gitelman, 2001; Small et al., 2001; Vandenberghe, Gitelman, Parrish, \& Mesulam, 2001; Wallace \& Stein, 1994; Warren, Zielin-

This research was supported by a Royal Society Collaboration Grant awarded to S.M.W. and K. Schill (Munich). Correspondence concerning this article should be addressed to S. M. Wuerger, Centre of Cognitive Neuroscience, Department of Psychology, Eleanor Rathbone Building, Bedford Street South, University of Liverpool, Liverpool L69 7ZA, England (e-mail: s.m.wuerger@liverpool.ac.uk). ski, Green, Rauschecker, \& Griffiths, 2002). The response rate of multisensory neurones in the SC is significantly enhanced when the sources of sensory input are co-localized and coincident in more than a single modality (King, Schnupp, \& Thompson, 1998; Stein, Huneycutt, \& Meredith, 1988; Wallace, Wilkinson, \& Stein, 1996). This response enhancement (Meredith \& Stein, 1983) is multiplicative and most pronounced when the unimodal stimuli are near threshold or ambiguous. Meredith and Stein (1996) refer to this as "inverse effectiveness," and in human psychophysics terms, cross-modal integration should cause a significant sensitivity enhancement. Some of the physiological findings are mirrored in behavioral performance: Behavioral studies show numerous cross-modal links in endogenous and exogenous spatial attention (Driver \& Spence, 1998a, 1998b; Lewald, Ehrenstein, \& Guski, 2001; Stein \& Meredith, 1993) for coincident (Spence \& Driver, 1994, 1996, 1997) and sequential (McDonald, Teder-Sälejärvi, \& Hillyard, 2000; Watanabe \& Shimojo, 1998 ) stimuli in the auditory and visual modalities. From an ecological point of view, it is likely that the human sensory system integrates not only positional cues, but also other cues that can be represented in multiple modalities, such as coherent motion. For moving objects, auditory and visual motion signals are correlated and provide information about the speed and the direction of the moving object; in addition, motion signals are powerful cues by which to infer the direction of heading (Gibson, 1957). The SC contains neurones that respond selectively to motion signals and are responsible for eye movements (Brecht, Goebel, Singer, \& Engel, 2001; Harris, Blakemore, \& Donaghy, 
1980; King et al., 1998; Meredith \& Stein, 1983; Stein \& Meredith, 1993; Wallace, McHaffie, \& Stein, 1997; Wallace, Meredith, \& Stein, 1998; Wallace \& Stein, 1997). If the processing of motion signals is analogous to that for positional cues, one might expect to see behavioral responses to cross-modal motion signals that are analogous to the data seen for co-localized signals. There is now behavioral evidence that auditory and visual motion signals can interact for suprathreshold conditions (Aspell, Bramwell, \& Hurlbert, 2000; Meyer \& Wuerger, 2001; Sekuler, Sekuler, \& Lau, 1997; Shams, Kamitani, \& Shimojo, 2000; Watanabe \& Shimojo, 2001). Suprathreshold auditory motion (Meyer \& Wuerger, 2001) introduces a strong response bias in visual motion detection - that is, a randomdot kinematogram (RDK) of $0 \%$ coherence is perceived as moving to the right (left) if it is accompanied by a suprathreshold auditory rightward (leftward) motion. This response bias is robust and occurs even if the auditory and the visual motion signals come from different sources and move at different speeds (Meyer \& Wuerger, 2001). It is still unclear whether auditory and visual motion integration also increases the sensitivity for motion detection, as opposed to a pure response bias. The aim of the present study was (1) to investigate how auditory and visual motion signals interact at threshold, (2) to investigate whether the presence of both visual and auditory motion signals increases the sensitivity for motion detection, and (3) to assess whether these audiovisual integration mechanisms are direction selective - that is, to test whether audiovisual motion integration is restricted to ecologically valid motion signals (same motion direction in both modalities).

\section{METHOD}

Audiovisual motion integration in human subjects was examined by measuring the motion detection thresholds when the motion was either visual or auditory motion or motion in both modalities.

\section{Apparatus}

The visual stimuli were generated using the Visual Stimulus Generator 2/5 (Cambridge Research Systems), which was controlled by a Dell Optiplex GX110 PC. The stimuli were then displayed on a large projection screen with a Hitachi LCD projector with a resolution of $800 \times 600$ pixels. The VSG system was connected to a RP2.1 Signal Processor (Tucker Davis) and HB7 Headphone Buffer, which generated the auditory stimuli. All the experiments were carried out in a darkened soundproof booth (IAC 1204A). The loudspeakers were mounted immediately behind the acoustically transparent projection screen and were separated by $7.4^{\circ}$, centered on the midpoint of the projection screen. The observer sat in front of the projection screen at a distance of $200 \mathrm{~cm}$.

\section{Stimuli}

The visual motion stimulus consisted of an RDK (Snowden \& Braddick, 1989), a black square $\left(16^{\circ}\right.$ of visual angle) in which 500 white dots (each dot subtended $0.05^{\circ}$ of visual angle) moved from a random start position along a line trajectory in a random direction. The proportion of dots that moved in a coherent direction could be varied to yield the percept of directed motion. Figure 1A is a schematic diagram showing the proportion of dots that move coherently to the right, varying from 0 (all dots move in random directions) to 1 (all dots move in the same direction). All dots moved at a fixed speed of $10 \mathrm{deg} / \mathrm{sec}$. To minimize the incidence of saccades in response to the visual stimulus (Westheimer, 1954), the duration of the RDK was set to $175 \mathrm{msec}$, during which six frames were presented. Using a constant-stimul us method, the proportion of coherently moving dots was varied between 0 and .32 . These coherence parameters had been established in a previous study (Meyer \& Wuerger, 2001).
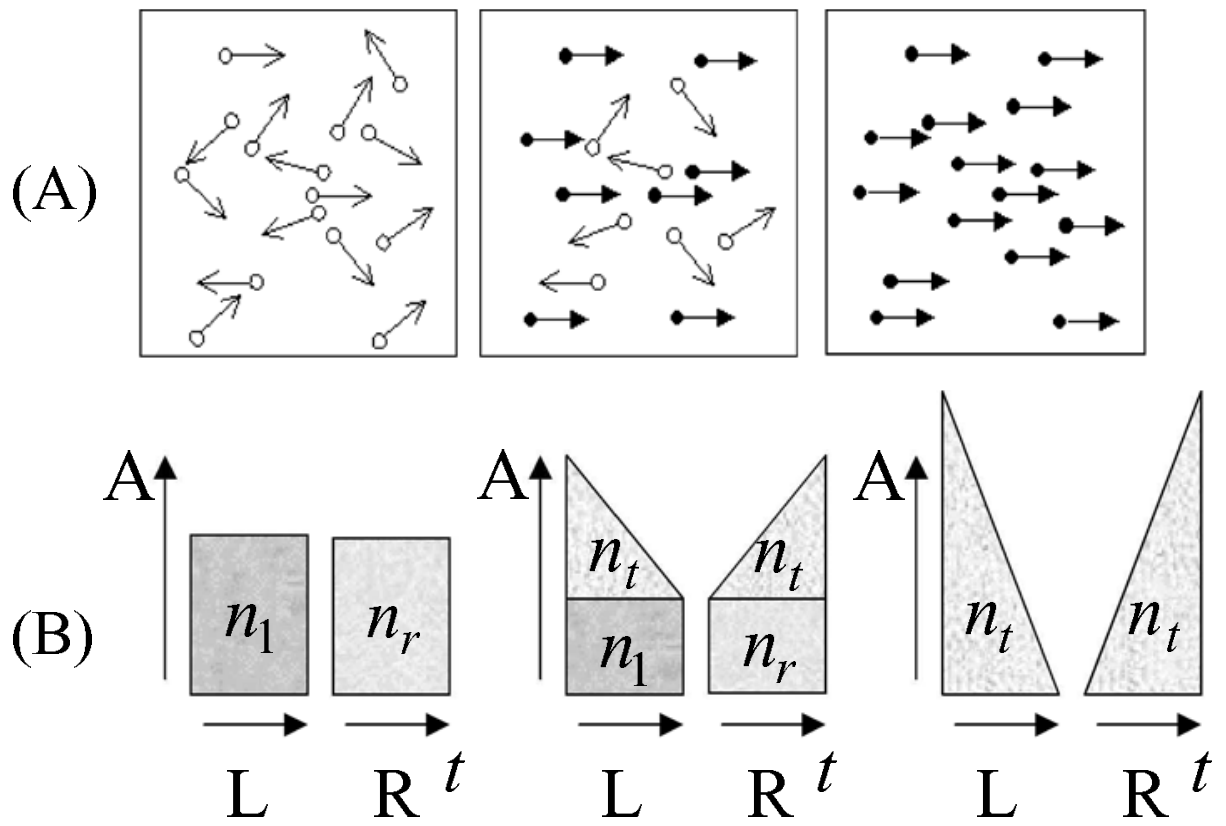

Figure 1. Visual and auditory motion stimuli. (A) The visual motion stimulus was a random-dot kinematogram. The relative number of dots moving in one direction (left or right) could be varied between $0(0 \%$ coherence $=$ noise $)$ and 1 . (B) The auditory motion stimulus was a white noise crossfaded between the two loudspeakers. The auditory coherence could be varied from 0 to 1. 
Similarly, auditory motion signals were created by changing the signal-to-noise ratio of white noise. The auditory motion signal was presented for 1,000 msec. At each trial, a pedestal white noise (noise) was presented that was different for the two loudspeakers (as is shown in Figure 1B). An incremental noise (signal), which was identical for the two loudspeakers, was added. This incremental identical noise was then cross-faded between the two loudspeakers to generate a moving acoustic signal. Sounds were presented at an average level of $65 \mathrm{~dB}(\mathrm{~A})$, measured at the listener position (Brüel \& Kjaer 2610 amplifier and 4133 microphone). The coherence levels used for the auditory signals ranged from 0 to .64; this range was established in a pilot study. The amplitude of the auditory noise was randomly jittered so that the observers could not use the amplitude as a cue for deciding which interval contained the coherent motion.

Previous studies have shown that visual target detection is affected when the visual target is preceded by an auditory cue with a delay below $300 \mathrm{msec}$ (Schmitt, Postma, \& de Haan, 2000). To avoid cuing effects due to the auditory motion onset, the visual stimulus onset was delayed by $412.5 \mathrm{msec}$, relative to the auditory stimulus onset. Hence, each trial lasted $1,000 \mathrm{msec}$ and the timing of the auditory and visual stimuli was as follows: onset of the auditory stimuli, 412.5-msec delay, onset of the visual stimulus, 412.5 delay, offset of the auditory and visual stimuli.

\section{Procedure}

In each experiment, all possible combinations of auditory and visual coherence levels were presented in a random sequence. For each modality, we used seven different coherence levels (three to the left, three to the right, and zero coherence), yielding 49 possible stimulus combinations. Each stimulus combination was presented four times in each experimental session, and each observer ran eight experimental sessions. Hence, the total number of trials run by each observer was $49 \times 4 \times 8=1,568$. The auditory and visual motion could be either consistent (i.e., both moving to the left or both to the right) or inconsistent (i.e., one moving to the right while the other moves to the left). The visual and the auditory stimuli could either be random (zero coherence $=$ noise) or have a small coherent motion in one of the two directions (visual coherence [ $\mathrm{coh}_{\mathrm{vis}}$ ] ranging from 0 to .32 and auditory coherence [ $\left[\mathrm{coh}_{\text {aud }}\right]$ ranging from 0 to .64). This allowed us to estimate the motion detection thresholds for each modality and for the combined audiovisual stimuli.

The subjects were instructed to fixate a cross that was presented continuously at the center of the visual stimulus. We used a two interval forced-choice (2IFC) paradigm: Each trial consisted of two intervals; one interval contained noise only $\left(\mathrm{coh}_{\mathrm{vis}}=\mathrm{coh}_{\mathrm{aud}}=0\right)$, whereas the other interval (signal) contained a visual or an auditory motion signal ( $\mathrm{coh}_{\mathrm{vis}}>0, \mathrm{coh}_{\mathrm{aud}}>0$, or both). The observer's task was to identify which interval contained any motion by pressing one of the two buttons on a button box. The response was valid only when the stimulus presentation in both intervals had been completed. The observers were instructed to attend to both modalities and report only the presence of motion, but not the direction of motion. An initial short training session was run before the main session in order to familiarize the observers with the apparatus and the task. Six subjects ( 3 males, 3 females), ranging from 20 to 40 years of age, took part in the experiment. All had normal or corrected-tonormal vision and showed no abnormalities in a clinical audiogram. The experiments followed the APA guidelines for experimentation with humans and were approved by the local ethics committee.

\section{Models}

To test our hypotheses about audiovisual integration and its direction selectivity, we fit two different models to the data. The two models differ in the assumed locus of interaction between auditory and visual motion signals. Model 1 assumes that representations of motion in the auditory and the visual stimuli are summed before a decision about the presence of motion is reached (subthreshold summation model), whereas Model 2 assumes that the auditory and the visual stimuli are processed independently in both modalities and that representations of local decisions are then summed up to reach a decision about the presence of a motion signal (probability summation model). Both models assume that, for the detection of bimodal motion, the signals from both modalities are combined without regard to sign - that is, independently of the direction of the motion signals. If this assumption is wrong, the fits for the consistent and the inconsistent conditions will differ from each other.

A Weibull function (Quick, 1974; Weibull, 1951) was fitted to estimate the threshold and the nonlinearity for each of the two modalities. The probability of detecting the visual signal is given by

$$
P\left(x_{\text {vis }}\right)=1-e^{-\left(\frac{x_{\text {vis }}}{\alpha_{\text {vis }}}\right)^{\beta_{\text {vis }}}},
$$

where $x_{\mathrm{vis}}$ is the coherence level of the visual motion stimulus and ranges from 0 to $1, \alpha_{\text {vis }}$ is the visual threshold value (corresponding to $81 \%$ correct responses), and $\beta_{\text {vis }}$ is the nonlinearity. An analogous equation describes the detection of the auditory signal, where $x_{\text {aud }}$ is the auditory coherence level (from 0 to 1 ), $\alpha_{\text {aud }}$ is the auditory threshold value, and $\beta_{\text {aud }}$ is the nonlinearity.

For stimuli that contain both a visual and an auditory motion signal, we tested two hypotheses as to how the auditory and the visual signals would be combined: subthreshold summation (Model 1) and probability summation (Model 2).

Model 1. The subthreshold summation model assumes that the auditory and the visual motion signals are summed before a decision about the presence of the joint motion is made. Hence, the probability that the joint stimulus will be detected is given by

$$
P\left(x_{\text {vis }}, x_{\text {aud }}\right)=1-e^{-\left(\frac{x_{\text {vis }}}{\alpha_{\text {vis }}}+\frac{x_{\text {aud }}}{\alpha_{\text {aud }}}\right)^{\beta}} .
$$

Since we used a IFC paradigm, we needed to take into account the chance performance $(\gamma)$-that is, .5-for two alternatives. For the Weibull function, the chance performance is the lower asymptote; the upper asymptote is set to 1 , since we can assume that the probability of a correct response converges to 1 for very large coherence levels. We can then express the probability of a correct response in a 2IFC task when an audiovisual stimulus $\left(x_{\mathrm{vis}}, x_{\text {aud }}\right)$ is presented as follows:

$$
P_{2 \mathrm{IFC}}\left(x_{\mathrm{vis}}, x_{\text {aud }}\right)=1-\gamma \cdot e^{-\left(\frac{x_{\text {aud }}}{\alpha_{\text {aud }}}+\frac{x_{\text {vis }}}{\alpha_{\text {vis }}}\right)^{\beta}} .
$$

We have three free parameters to estimate in Model 1.

Model 2. Model 2 assumes that the two motion signals are processed independently and the joint detection performance is based on the independent decisions derived in the two modalities (probability summation; Quick, 1974). The probability that the joint stimulus will not be detected is then given by the probability that neither the visual nor the auditory system will detect the stimulus. Hence, the probability that the joint stimulus will be detected is given by

$$
P\left(x_{\text {vis }}, x_{\text {aud }}\right)=1-\left[1-P\left(x_{\text {vis }}\right)\right]\left[1-P\left(x_{\text {aud }}\right)\right] .
$$

From Equations 1 and 4, it follows that

$$
P\left(x_{\mathrm{vis}}, x_{\mathrm{aud}}\right)=1-e^{-\left[\left(\frac{x_{\mathrm{vis}}}{\alpha_{\mathrm{vis}}}\right)^{\beta_{\mathrm{vis}}}+\left(\frac{x_{\text {aud }}}{\alpha_{\text {aud }}}\right)^{\beta_{\text {aud }}}\right]} .
$$

Since we used a 2IFC paradigm, we needed to take into account the chance performance $(\gamma)$ and the probability of a correct response in a 2IFC task when an audiovisual stimulus $\left(x_{\text {vis }}, x_{\text {aud }}\right)$ is given by

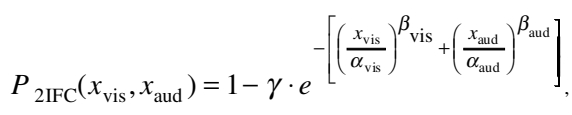

where $\alpha_{\text {vis }}$ and $\alpha_{\text {aud }}$ are the respective threshold values and correspond to the coherence level at which the motion is detected with a 
probability of .81 . If we assume that the nonlinearities $\left(\beta_{\text {aud }}, \beta_{\text {vis }}\right)$ are identical in both modalities, we need to estimate three free parameters from our data - namely, the thresholds in both modalities and the nonlinearity. We will refer to Equation 6 with $\beta_{\text {aud }}=\beta_{\text {vis }}$ as Model 2.

\section{RESULTS}

To assess whether auditory and visual motion signals are linearly summed before a decision about the presence of a motion signal is made (subthreshold summation) and whether this integration depends on the motion direction of the auditory and the visual signals, we measured motion sensitivity for the following two conditions: both the auditory and the visual motions were to the left or to the right (consistent motion) or the auditory and the visual motions were in different directions (inconsistent motion-i.e., visual to the right and auditory to the left or vice versa). If the integration between auditory and visual

A

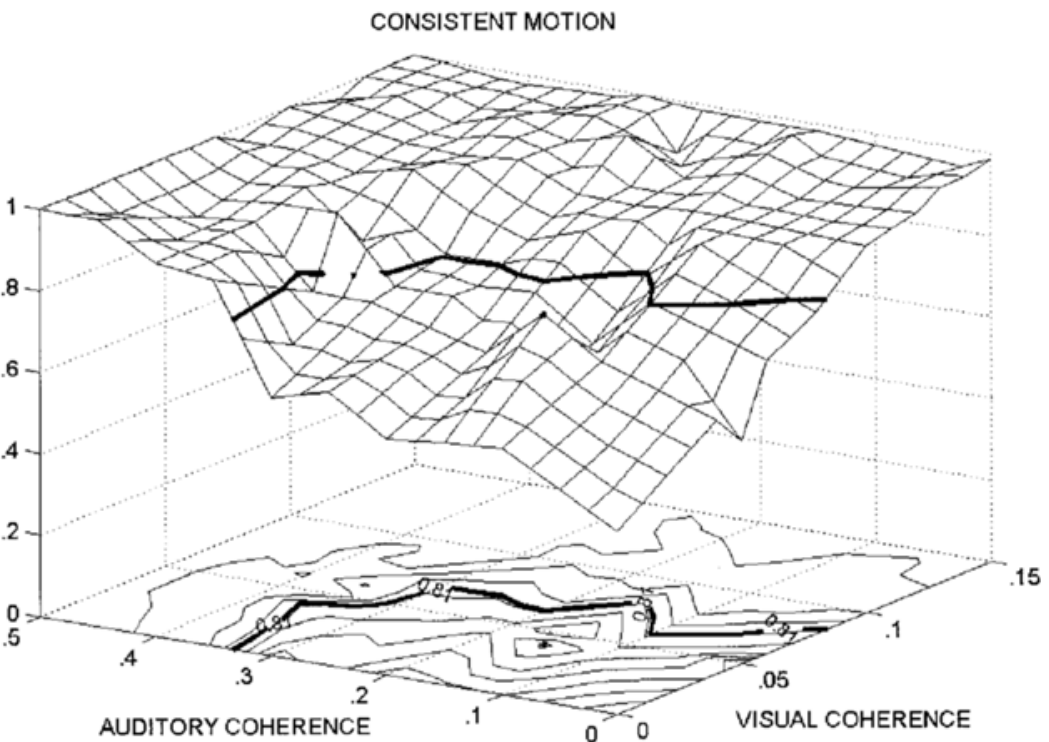

B INCONSISTENT MOTION

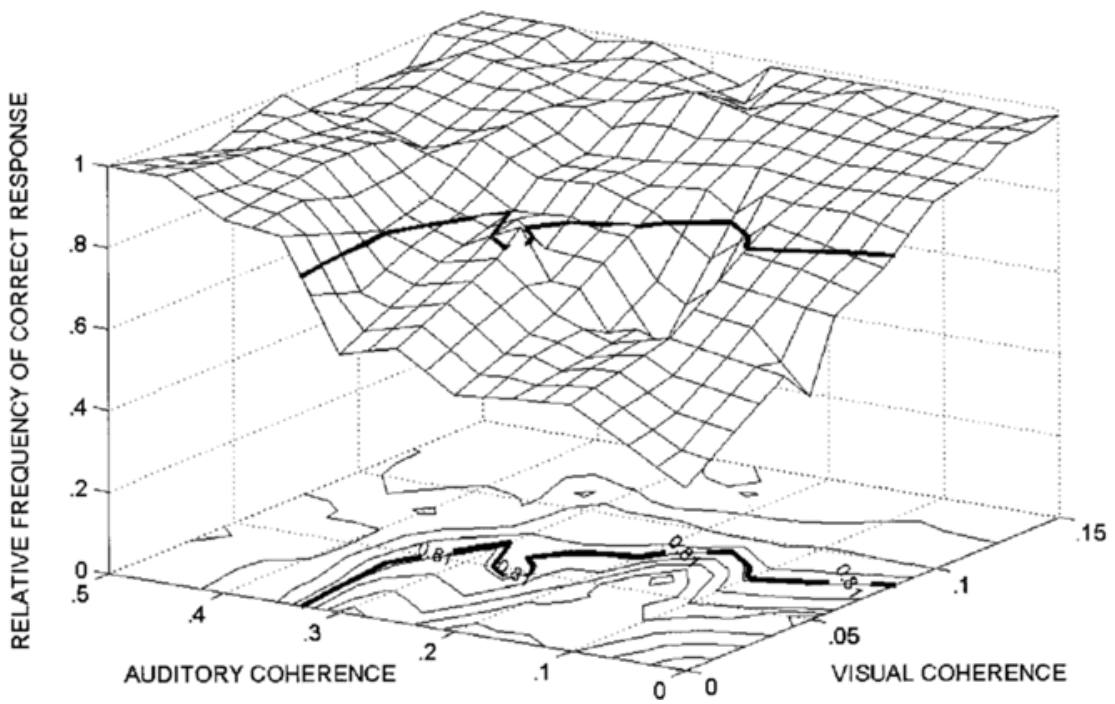

Figure 2. Two-dimensional psychometric functions (averaged over all 6 observers) for consistent (A) and inconsistent (B ) motion signals. The relative frequency of a correct response (motion detection) is plotted as a function of the auditory and the visual coherence of the motion signal. Chance performance is at .5. The contour plot in the $x, y$ plane of the graph shows lines of equal performance. The thick lines indicate threshold contours-that is, the audiovisual motion coherence levels at which the observers responded correctly in $81 \%$ of all cases. 
motion signals is direction blind, we would expect similar results for the consistent and the inconsistent motion conditions. Figure 2 shows the relative frequency of a correct motion response for both the consistent (Figure 2A) and the inconsistent (Figure 2B) motion conditions as a function of the auditory and the visual coherence levels, averaged over all 6 observers. The thick lines indicate the threshold contour - that is, that audiovisual motion coherence at which the observers responded correctly in $81 \%$ of the cases. The threshold contours are shown in both the contour plot and the two-dimensional psychometric function graph. For both consistent and inconsistent motion conditions, the threshold contour forms a rectangle with a rounded-off corner.

These surfaces are empirical two-dimensional psychometric functions with two independent variables (auditory and visual motion coherence) and one dependent variable (correct responses in the motion task). To establish the nature of audiovisual integration and its direction selectivity, we fit two different models to the two-dimensional psychometric functions (see the Method section for details). Model 1 (subthreshold summation) assumes that the auditory and the visual motion signals are linearly summed up (without regard for the direction of the motion) before a decision about the presence of motion is reached; Model 2 (probability summation) assumes that the two signals are combined after they have been processed in both modalities. We fit both models to our data by minimizing the squared deviations between the model predictions and the data points. To compare the goodness of the model fits, we computed the squared deviations that follow a chi-square distribution with $(n-m)$ degrees of freedom, where $n$ is the total number of different data points and $m$ is the number of estimated parameters (Lienert, 1962). Table 1 contains the chi-square test statistics for both models and for both stimulus conditions (consistent vs. inconsistent motion), the corresponding degrees of freedom, and the resulting error probabilities $(p)$ for each observer and for both conditions (inconsistent vs. consistent). The probability of rejecting the model given that the underlying model is correct is denoted with $p$; a small value for $p$ indicates a poor model fit. The $p$ values are identical for both motion conditions (consistent vs. inconsistent auditory and visual motion): .035 (Model 1) and .301 (Model 2) for consistent motion and .007 (Model 1) and .307 (Model 2) for inconsistent motion. For both motion conditions, Model 2 fits the data better than does Model 1. Error probabilities below 1 indicate a lack of fit or a very poor fit (Lienert, 1962); the subthreshold summation model therefore cannot account for the perceptual data. The probability summation model cannot be rejected and provides a satisfactory fit for the consistent and the inconsistent motion conditions ( $p=.301$ and .307 , respectively).

Figure 3 shows the predicted psychometric functions for the subthreshold summation model (A) and the probability summation model (B). The major qualitative difference is that subthreshold summation predicts that the thresholds for intermediate audiovisual directions lie on a straight line; the thick lines in Figure 3 indicate the predicted threshold contours - that is, the audiovisual motion coherences at which a performance of $81 \%$ correct responses is predicted. Probability summation predicts that the threshold detection contours are not straight but curved, which is again indicated by the thick lines in the contour and the three-dimensional predictions. Comparing the observed threshold contours (thick lines in Figures $2 \mathrm{~A}$ and $2 \mathrm{~B}$ ) with the predicted threshold contours (thick lines in Figures 3A and 3B) shows that the probability summation model fits the data, whereas subthreshold summation provides a poor fit to the data. This is confirmed by the statistical tests (Table 1). Since the predictions for inconsistent and consistent motion are almost identical (Table 2), only the predictions for consistent motion are shown in Figures 3A and 3B.

The estimated auditory and visual thresholds, as well as the nonlinearities, for both models and for all observers are shown in Table 2. The mean auditory threshold $\left(\alpha_{\text {aud }}\right)$ corresponding to the $81 \%$ correct is about $35 \%-46 \%$ coherence, and the mean visual threshold $\left(\alpha_{\mathrm{vis}}\right)$ is about

Table 1

Goodness of Fit for Both Models

\begin{tabular}{|c|c|c|c|c|c|c|c|c|c|c|c|c|}
\hline \multirow[b]{3}{*}{ Observer } & \multicolumn{6}{|c|}{ Consistent Motion } & \multicolumn{6}{|c|}{ Inconsistent Motion } \\
\hline & \multicolumn{3}{|c|}{$\begin{array}{l}\text { Subthreshold } \\
\text { Summation }\end{array}$} & \multicolumn{3}{|c|}{$\begin{array}{l}\text { Probability } \\
\text { Summation }\end{array}$} & \multicolumn{3}{|c|}{$\begin{array}{l}\text { Subthreshold } \\
\text { Summation }\end{array}$} & \multicolumn{3}{|c|}{$\begin{array}{l}\text { Probability } \\
\text { Summation }\end{array}$} \\
\hline & $\chi^{2}$ & $d f$ & $p$ & $\chi^{2}$ & $d f$ & $p$ & $\chi^{2}$ & $d f$ & $p$ & $\chi^{2}$ & $d f$ & $p$ \\
\hline 1 & 30.3 & 20 & .066 & 22.7 & 21 & .358 & 33.9 & 19 & .019 & 25.6 & 19 & .141 \\
\hline 2 & 45.4 & 22 & .002 & 22.8 & 19 & .244 & 47.5 & 22 & .001 & 24.9 & 22 & .297 \\
\hline 3 & 28.8 & 20 & .091 & 29.7 & 21 & .097 & 41.9 & 23 & .009 & 29.6 & 22 & .127 \\
\hline 4 & 34.2 & 26 & .129 & 24.4 & 24 & .438 & 33.7 & 24 & .089 & 22.1 & 21 & .393 \\
\hline 5 & 34.4 & 10 & .001 & 9.9 & 12 & .621 & 36.4 & 18 & .006 & 16.1 & 15 & .373 \\
\hline 6 & 29.4 & 12 & .003 & 25.0 & 13 & .023 & 36.4 & 12 & .001 & 13.7 & 12 & .317 \\
\hline Median & & & .035 & & & .301 & & & .007 & & & .307 \\
\hline
\end{tabular}

Note-The chi-square distributed test statistics, the degrees of freedom, and the resulting error probabilities are shown for both models and for both motion conditions. Consistent and inconsistent motion detection yielded similar fits. The subthreshold summation model must be rejected for both motion conditions. The probability summation model provides a satisfactory fit for the inconsistent and the consistent motion conditions. 


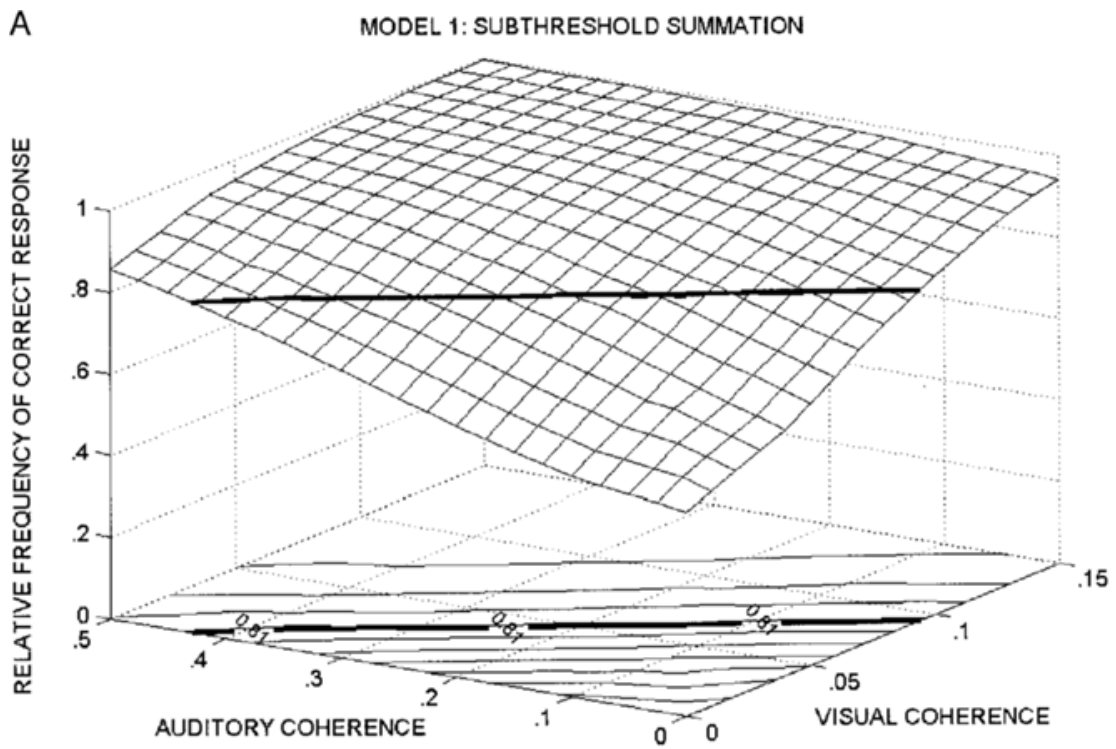

B MODEL 2: PROBABILITY SUMMATION

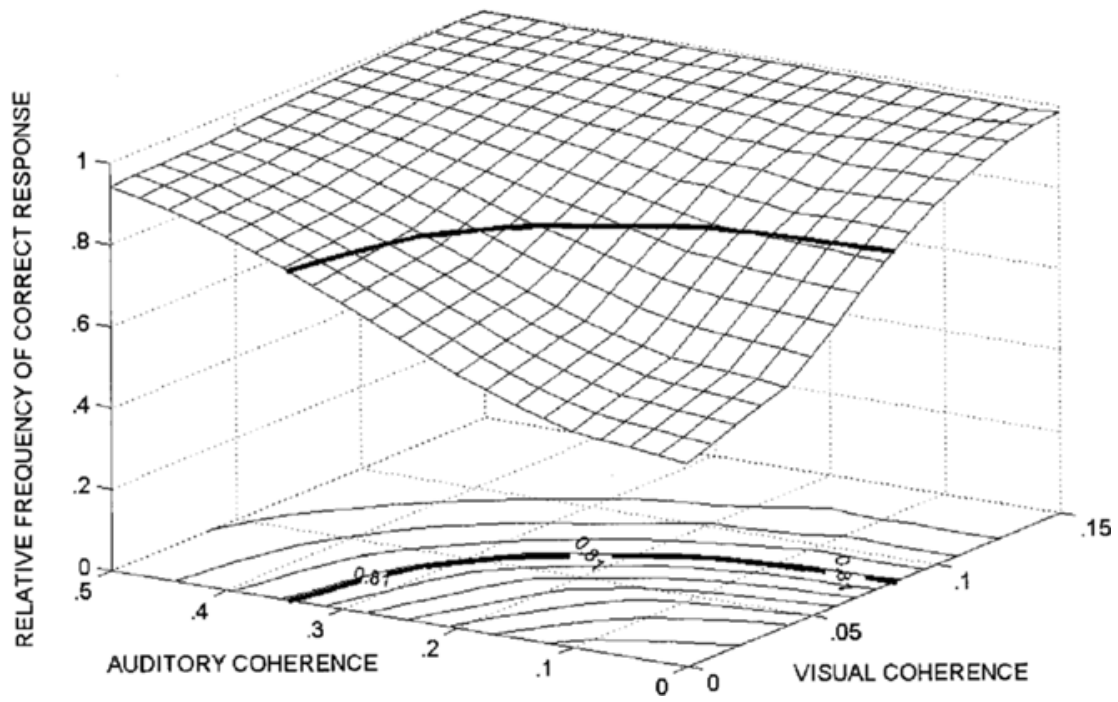

Figure 3. Two-dimensional predictions for (A) the subthreshold summation model and (B) the probability summation model. Subthreshold summation predicts that the thresholds for stimuli that contain both auditory and visual motion signals lie on a straight line in the contour plot. Probability summation predicts larger thresholds for the intermediate audiovisual motion stimuli. The predictions for inconsistent and consistent motion are almost identical (cf. Table 2); hence, only the predictions for consistent motion are shown. The contour lines are lines of equal performance in the motion task. The thick lines indicate the predicted threshold contours-that is, the $81 \%$ correct contour.

$8 \%-10 \%$ coherence, which is in good agreement with previous studies. It is known that motion coherence sensitivity depends on a variety of parameters, such as stimulus duration, dot size, dot density, speed, and spatial frequency (e.g., Barlow \& Tripathy, 1997; Braddick, 1997; Burr \& Santoro, 2001; Grossman \& Blake, 1999; Gurney \& Wright, 1996; Meyer \& Wuerger, 2001; Scase, Braddick, \& Raymond, 1996; Smith, Snowden, \& Milne, 1994).
Burr and Santoro demonstrated that motion coherence sensitivity depends strongly on stimulus duration. For stimulus durations comparable to ours $(175 \mathrm{msec})$, they reported motion coherence thresholds of about $10 \%$, which is in agreement with our findings. In our experiments, we found no difference between the consistent and the inconsistent motion conditions. The nonlinearity $(\beta)$ for the probability summation model was about $2.2-2.4$ 
Table 2

Estimated Thresholds for Both Models

\begin{tabular}{|c|c|c|c|c|c|c|c|c|c|c|c|c|}
\hline \multirow[b]{3}{*}{ Observer } & \multicolumn{6}{|c|}{ Consistent Motion } & \multicolumn{6}{|c|}{ Inconsistent Motion } \\
\hline & \multicolumn{3}{|c|}{$\begin{array}{l}\text { Subthreshold } \\
\text { Summation }\end{array}$} & \multicolumn{3}{|c|}{$\begin{array}{l}\text { Probability } \\
\text { Summation }\end{array}$} & \multicolumn{3}{|c|}{$\begin{array}{c}\text { Subthreshold } \\
\text { Summation }\end{array}$} & \multicolumn{3}{|c|}{$\begin{array}{l}\text { Probability } \\
\text { Summation }\end{array}$} \\
\hline & $\alpha_{\text {aud }}$ & $\alpha_{\mathrm{vis}}$ & $\beta$ & $\alpha_{\text {aud }}$ & $\alpha_{\mathrm{vis}}$ & $\beta$ & $\alpha_{\text {aud }}$ & $\alpha_{\mathrm{vis}}$ & $\beta$ & $\alpha_{\text {aud }}$ & $\alpha_{\mathrm{vis}}$ & $\beta$ \\
\hline 1 & .40 & .08 & 1.5 & .35 & .07 & 1.8 & .40 & .09 & 1.6 & .35 & .07 & 2.0 \\
\hline 2 & .42 & .10 & 1.7 & .33 & .08 & 2.9 & .41 & .10 & 1.60 & .32 & .08 & 2.6 \\
\hline 3 & .42 & .09 & 2.0 & .37 & .07 & 2.0 & .47 & .09 & 1.7 & .39 & .08 & 2.1 \\
\hline 4 & .67 & .12 & 1.7 & .40 & .11 & 2.2 & .73 & .11 & 1.8 & .41 & .11 & 2.5 \\
\hline 5 & .36 & .08 & 1.8 & .35 & .07 & 2.7 & .37 & .08 & 1.2 & .36 & .08 & 2.7 \\
\hline 6 & .33 & .10 & 1.9 & .30 & .09 & 1.5 & .36 & .12 & 1.9 & .29 & .10 & 2.5 \\
\hline Mean & .43 & .10 & 1.7 & .35 & .08 & 2.2 & .46 & .10 & 1.7 & .36 & .09 & 2.4 \\
\hline
\end{tabular}

Note-The auditory $\left(\alpha_{\text {aud }}\right)$ and visual $\left(\alpha_{\text {vis }}\right)$ thresholds, as well as the nonlinearity $(\beta)$, are estimated for both models and both motion conditions. There is no significant difference in the estimated parameters for the consistent and the inconsistent motion conditions.

and was in accordance with previous studies (Meyer \& Wuerger, 2001).

A third model assuming completely independent decisions in the auditory and the visual pathways and complete lack of summation predicts that the detection contours shown in Figures 3A and 3B would form rectangles. This model can be easily rejected, since it is a special case of Model 2 with $\beta$ going to infinity; the best fitting $\beta$ is about 2.2-2.4. The shape of the detection contours (not rectangular but rounded) suggests that the observers based their decision about the presence of motion on both modalities, and not only on the auditory or only on the visual stimuli.

\section{DISCUSSION}

Our aim was to characterize the locus and the nature of audiovisual motion integration mechanisms and to assess whether audiovisual integration is constrained to congruous motion in the two modalities. Our first major finding is that our data are consistent with the hypothesis that the auditory and the visual motion signals are integrated according to a simple probability summation rule: The signals are processed independently and then combined at a decision level. We are aware of only one study that tested both models (subthreshold summation vs. probability summation models) for the integration of auditory and visual stimuli (Mulligan \& Shaw, 1980): Spatially coincident auditory and visual pulses embedded in auditory and visual noise were used. The observers had to report the presence of the signal, which could be visual, auditory, or bimodal. The conclusion from that experiment was that probability summation predicts audiovisual integration performance better than the subthreshold summation model does. Their experiment tested the integration of spatially coincident, stationary auditory and visual pulses, whereas we have investigated the integration of auditory and visual motion signals. Nonetheless, we reached the same conclusionnamely, that the early processing of auditory and visual stimuli occurs, to a large extent, independently and that the output of the modalities is then optimally combined following a simple probability summation rule. Similarly, more recent results (Alais \& Burr, 2001) have suggested that the integration of auditory and visual motion stimuli is governed by a simple probability summation rule, and not by early subthreshold summation.

Our results seem to be at odds with neurophysiological recordings from neurons in the SC (Brecht et al., 2001; Meredith \& Stein, 1983; Rauschecker, 1988; Rauschecker \& Harris, 1989; Stein \& Meredith, 1993; Wallace et al., 1997; Wallace et al., 1998; Wallace \& Stein, 1997), which show integration at the sensory level that are more consistent with subthreshold summation or even response enhancement. Neurones in the SC are known to be motion sensitive and to control eye movements but are not directly implicated in conscious motion perception. Hence, we expect that subthreshold summation of auditory and visual stimuli may occur in tasks in which SC neurones play a major role, such as in gaze control.

Our second finding is that this integration mechanism does not depend on the direction of the auditory and visual motion signals and also occurs for incongruous motion signals. The fact that this integration is direction blind is in accordance with the first finding-namely, that the integration occurs after basic auditory and visual motion processing has occurred. This is consistent with recent fMRI studies, which have identified cortical sites (parietal cortex) that can be activated by simultaneous auditory and visual motion inputs (Bremmer, Schlack, Shah, et al., 2001; Rauschecker, 1988; Rauschecker \& Harris, 1989). We speculate that early audiovisual motion integration may play a major role in the control of eye movement and gaze, whereas late integration mechanisms are involved in conscious motion perception.

\section{REFERENCES}

Alais, D., \& Burr, D. (2001). No low-level interaction between visual and auditory motion [Abstract]. Perception, 30(Suppl.), 5.

Aspell, J. E., Bramwell, D. I., \& Hurlbert, A. C. (2000). Interactions between visual and auditory movement perception in a direction discrimination task. Perception, 29(Suppl.), 74.

Barlow, H., \& Tripathy, S. P. (1997). Correspondence noise and signal pooling in the detection of coherent visual motion. Journal of Neuroscience, 17, 7954-7966.

BRADDICK, O. (1997). Local and global representations of velocity: 
Transparency, opponency, and global direction perception. Perception, 26, 995-1010.

Brecht, M., Goebel, R., Singer, W., \& Engel, A. K. (2001). Synchronisation of visual responses in the superior colliculus of awake cats. NeuroReport, 12, 43-47.

Bremmer, F., Schlack, A., Duhamel, J. R., Graf, W., \& Fink, G. R. (2001). Space coding in primate posterior parietal cortex. NeuroImage, 14, S46-S51.

Bremmer, F., Schlack, A., Shah, N. J., Zafiris, O., Kubischik, M., Hoffmann, K. P., Zilles, K., \& Fink, G. R. (2001). Polymodal motion processing in posterior parietal and promotor cortex: A human fMRI study strongly implies equivalencies between humans and monkeys. Neuron, 29, 287-296.

BurR, D. C., \& SAntoro, L. (2001). Temporal integration of optic flow, measured by contrast and coherence thresholds. Vision Research, 41, 1891-1899.

Calvert, G. A., Brammer, M. J., Bullmore, E. T., Campbell, R, Iverson, S. D., \& David, A. S. (1999). Response amplification in sensory-specific cortices during cross-modal binding. NeuroReport, 10, 2619-2623.

Calvert, G. A., Bullmore, E. T., Brammer, M. J., Campbell, R. Williams, S. C., McGuire, P. K., Woodruff, P. W., Iversen, S. D., \& DAvID, A. S. (1997). Activation of auditory cortex during silent lipreading. Science, 276, 593-596.

Daffner, K. R., Mesulam, M. M., Scinto, L. F. M., Acar, D., Calvo, V., Faust, R. Chaberic, A., Kennedy, B., \& Holcomb, P. (2000). The central role of the prefrontal cortex in directing attention to novel events. Brain, 123, 927-939.

Driver, J., \& Spence, C. (1998a). Attention and the crossmodal construction of space. Trends in Cognitive Sciences, 2, 254-263.

Driver, J., \& SPENCE, C. (1998b). Cross-modal links in spatial attention. Philosophical Transactions of the Royal Society of London: Series $B$, 353, 1319-1331.

Foxe, J. J., Morocz, I. A., Murray, M. M., Higgins, B. A., Javitt, D. C., \& Schroeder, C. E. (2000). Multisensory auditorysomatosensory interactions in early cortical processing revealed by high-density electrical mapping. Cognitive Brain Research, 10, 77-83.

Foxe, J. J., \& Simpson, G. V. (2002). Flow of activation from V1 to frontal cortex in humans: A framework for defining "early" visual processing. Experimental Brain Research, 142, 139-150.

Fu, K. M. G., Foxe, J. J., Murray, M. M., Higgins, B. A., Javitt, D. C., \& Schroeder, C. E. (2001). Attention-dependent suppression of distracter visual input can be cross-modally cued as indexed by anticipatory parieto-occipital alpha-band oscillations. Cognitive Brain Research, 12, 145-152.

Fuster, J. M., Bodner, M., \& Kroger, J. K. (2000). Cross-modal and cross-temporal association in neurons of frontal cortex. Nature, $\mathbf{4 0 5}$, 347-351.

Gibson, J. J. (1957). Optical motions and transformation as stimuli for visual perception. Psychological Review, 64, 288-295.

Gitelman, D. R., Parrish, T. B., Friston, K. J., \& Mesulam, M. M. (2002). Functional anatomy of visual search: Regional segregations within the frontal eye fields and effective connectivity of the superior colliculus. NeuroImage, 15, 970-982.

Griffiths, T. D., Rees, G., Rees, A., Green, G. G. R., Witton, C., Rowe, D., Buchel, C., Turner, R., \& Frackowiak, R. S. (1998). Right parietal cortex is involved in the perception of sound movement in humans. Nature Neuroscience, 1, 74-79.

Grossman, E. D., \& Blake, R. (1999). Perception of coherent motion, biological motion and form-from-motion under dim-light conditions. Vision Research, 39, 3721-3727.

Gurney, K. N., \& Wright, M. J. (1996). A model for the spatial integration and differentiation of velocity signals. Vision Research, 36, 2939-2955.

Harris, L. R., Blakemore, C., \& Donaghy, M. (1980). Integration of visual and auditory space in the mammalian superior colliculus. $\mathrm{Na}$ ture, 288, 56-59.

King, A. J., \& CAlvert, G. (2001). Multisensory integration: Perceptual grouping by eye and ear. Current Biology, 11, 322-325.

King, A. J., Schnupp, J. W. H., \& Thompson, I. D. (1998). Signals from the superficial layers of the superior colliculus enable the development of the auditory space map in the deeper layers. Journal of Neuroscience, 18, 9394-9408.

Lewald, J., Ehrenstein, W. H., \& Guski, R. (2001). Spatio-temporal constraints for auditory-visual integration. Behavioural Brain Research, 121, 69-79.

Lewis, J. W., Beauchamp, M. S., \& De Yoe, E. A. (2000). A comparison of visual and auditory motion processing in human cerebral cortex. Cerebral Cortex, 10, 873-888.

Lienert, G. A. (1962). Verteilungsfreie Verfahren in der Biostatistik. Meisenheim: A. Hain.

McDonald, J. J., Teder-SÄlejärvi, W. A., \& Hillyard, S. A. (2000). Involuntary orienting to sound improves visual perception. Nature, 407, 906-908.

Meredith, M. A., Nemitz, J. W., \& Stein, B. E. (1987). Determinants of multisensory integration in superior colliculus neurones: I. Temporal factors. Journal of Neuroscience, 10, 3215-3229.

Meredith, M. A., \& Stein, B. E. (1983). Interactions among converging sensory inputs in the superior colliculus. Science, 221, 389-391.

Meredith, M. A., \& Stein, B. E. (1996). Spatial determinants of multisensory integration in cat superior colliculus. Journal of Neurophysiology, 75, 1843-1857.

Mesulam, M. M., Nobre, A. C., Kim, Y. H., Parrish, T. B., \& GitelMAN, D. R. (2001). Heterogeneity of cingulate contributions to spatial attention. NeuroImage, 13, 1065-1072.

Meyer, G., \& Wuerger, S. (2001). Cross-modal integration of auditory and visual motion signals. NeuroReport, 12, 2557-2600.

Mulligan, R. M., \& SHaw, M. L. (1980). Multimodal signal detection: Independent decisions vs. integration. Perception \& Psychophysics, 28, 471-478.

Quick, R. F. (1974). A vector magnitude model of contrast detection. Kybernetik, 16, 65-67.

RAUSCHECKER, J. P. (1988). Visual function of the cat's LP/LS subsystem in global motion processing. Progress in Brain Research, 75, 95 108.

Rauschecker, J. P., \& Harris, L. R. (1989). Auditory and visual neurons in the cat's superior colliculus selective for the direction of apparent motion stimuli. Brain Research, 490, 56-63.

ScASE, M. O., BRAdDick, O. J., \& RAY mond, J. E. (1996). What is noise for the motion system? Vision Research, 36, 2579-2586.

Schmitt, M., Postma, A., \& DE HAAN, E. (2000). Interactions between exogenous auditory and visual spatial attention. Quarterly Journal of Experimental Psychology, 53, 105-130.

Sekuler, R., Sekuler, A. B., \& Lau, R. (1997). Sound alters visual motion perception. Nature, $\mathbf{3 8 5}, 308$.

Shams, L., Kamitani, Y., \& Shimojo, S. (2000). What you see is what you hear. Nature, $\mathbf{4 0 8}, 788$.

Small, D. M., Gitelman, D. R., Gregory, M. D., Nobre, A. C., ParRISH, T., \& Mesulam, M. M. (2001). The posterior cingulate cortex and anticipatory shifts of spatial attention. NeuroImage, 13, S360S360.

Smith, A. T., Snowden, R. J., \& Milne, A. B. (1994). Is global motion really based on spatial integration of local motion signals. Vision Research, 34, 2425-2430.

SNOWDEN, R. J., \& BRADDICK, O. J. (1989). The combination of motion signals over time. Vision Research, 29, 1621-1630.

Spence, C., \& Driver, J. (1994). Covert spatial orienting in audition. Journal of Experimental Psychology: Human Perception \& Performance, 20, 555-574.

Spence, C., \& Driver, J. (1996). Audiovisual links in endogenous covert spatial attention. Journal of Experimental Psychology: Human Perception \& Performance, 22, 1000-1030.

SPENCE, C., \& Driver, J. (1997). Audiovisual links in exogenous covert spatial orienting. Perception \& Psychophysics, 59, 1-22.

Stein, B. E., Huneycutt, W. S., \& Meredith, M. A. (1988). Neurons and behavior: The same rules of multisensory integration apply. Brain Research, 448, 355-358.

Stein, B. E., \& Meredith, M. A. (1993). The merging of the senses. Cambridge, MA: MIT Press.

Vandenberghe, R., Gitelman, D. R, Parrish, T. B., \& Mesulam, 
M. M. (2001). Functional specif icity of superior parietal mediation of spatial shifting. NeuroImage, 14, 661-673.

Wallace, M. T., McHaffie, J. G., \& Stein, B. E. (1997). Visual response properties and visuotopic representation in the newborn monkey superior colliculus. Journal of Neurophysiology, 78, 2732-2741.

Wallace, M. T., Meredith, M. A., \& Stein, B. E. (1998). Multisensory integration in the superior colliculus of the alert cat. Journal of Neurophysiology, 80, 1006-1010.

Wallace, M. T., \& STein, B. E. (1994). Cross-modal synthesis in the mid-brain depends on input from association cortex. Journal of Neurophysiology, 71, 429-432.

Wallace, M. T., \& Stein, B. E. (1997). Development of multisensory neurons and multisensory integration in cat superior colliculus. Journal of Neuroscience, 17, 2429-2444.

Wallace, M. T., Wilkinson, L. K., \& Stein, B. E. (1996). Representation and integration of multiple sensory inputs in primate superior colliculus. Journal of Neurophysiology, 76, 1246-1266.
Warren, J. D., Zielinski, B. A., Green, G. G. R., Rauschecker, J. P., \& GRIFFITHS, T. D. (2002). Perception of sound-source motion by the human brain. Neuron, 34, 139-148.

Watanabe, K., \& Shimojo, S. (1998). Attentional modulation in perception of visual motion events. Perception, 27, 1041-1054.

Watanabe, K., \& Shimojo, S. (2001). When sound affects vision: Effects of auditory grouping on visual motion perception. Psychological Science, 12, 109-116.

WeIBULL, W. (1951). A statistical distribution function of wide applicability. Journal of Applied Mechanics, 18, 292-297.

Westheimer, G. (1954). Eye movement responses to a horizontally moving stimulus. Archives of Ophthalmology, 52, 932-941.

(Manuscript received August 3, 2002;

revision accepted for publication April 12, 2003.) 